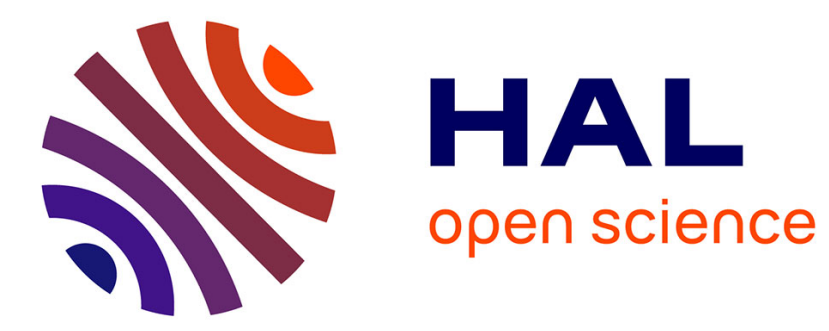

\title{
LE COLOSTRUM DE VACHE. Composition - Propriétés. RÉPERCUSSIONS EN INDUSTRIE LAITIÈRE
}

\author{
A. Houdinière
}

\section{- To cite this version:}

A. Houdinière. LE COLOSTRUM DE VACHE. Composition - Propriétés. RÉPERCUSSIONS EN INDUSTRIE LAITIÈRE. Le Lait, 1944, 24 (234_236), pp.108-139. hal-00927891

\section{HAL Id: hal-00927891 \\ https://hal.science/hal-00927891}

Submitted on 1 Jan 1944

HAL is a multi-disciplinary open access archive for the deposit and dissemination of scientific research documents, whether they are published or not. The documents may come from teaching and research institutions in France or abroad, or from public or private research centers.
L'archive ouverte pluridisciplinaire HAL, est destinée au dépôt et à la diffusion de documents scientifiques de niveau recherche, publiés ou non, émanant des établissements d'enseignement et de recherche français ou étrangers, des laboratoires publics ou privés. 
par celle de glycinine, étant entendu que ce mot ne doit s'appliquer qu'à une espèce chimique pure.

Quant aux mélanges plus ou moins variables et inconnus de protéines diverses que l'on peut extraire d'une graine de légumineuse quelconque, on devra conserver le terme général de légumine en lui accolant le nom de la plante d'où provient ce mélange : légumine du pois, du haricot, de la lentille, de la fève, du lupin, du soya, etc...

Ainsi on évitera toute confusion entre les divers produits purs ou impurs obtenus en partant de ces différentes graines.

\section{LE COLOSTRUM DE VACHE Composition - Propriétés RÉPERCUSSIONS EN INDUSTRIE LAITIÈRE} par

\section{A. HOUDINIÈRE}

Ingénieur agrieole

Docteur-Vétérinaire sanitaire du département de la Seine

\section{PLAN DU TRAVAII}

Nous croyons utile de donner une vue générale des principales questions traitées pour permettre au lecteur de s'orienter facilement vers les parties qui l'intéressent plus particulièrement.

\section{INTRODUCTION.}

GÉnÉralités.

CHAPITRE I. - ÉTUDE PHYSICO:CHIMIQUE DU COLOSTRUM.

A. Constitution pHysico-Chimique.

I. Composition chimique :

10 Généralités;

$2^{\circ}$ Composition du premier colostrum;

$3^{\circ}$ Evolution de la eomposition moyenne aú cours de la période colostrale ;

40 Etude chimique de quelques composants :

a) Eau et extrait sec ;

b) Lipides (glycérides, phosphoaminolipides, stérols);

c) Composés azotés (protidiques, non protidiques) :

d) Glucides (lactose et autres sucres);

e) Matières salines (éléments de base, oligoéléments).

II. Constitution physique :

10 La solution vraie (solution ionique, moléculaire);

$2^{\circ}$ La solution colloïdale (col. protéiniques, minéraux);

$3^{\circ}$ L'émulsion. 
B. Constantes physiques et ohimiques.

I. Constantes fixes :

a) Point de congélation ;

b) C. M. S.

II. Constantes variables :

a) Constantes physiques ;

b) Constantes chimiques.

C. Proprintés physico-ohimiques.

I. Action des agents physiques :

a) Du repos ;

b) De la force centrifuge ;

c) De la chaleur.

II. Action des agents chimiques :

a) De l'alcool ;

b) Des acides.

III. Action combinée des agents physiques et chimiques.

De la chaleur et des acides.

\section{CHAPITRE II. - ÉTUDE BIOLOGIQUE DU COLOSTRUM}

A. Constitution biologique.

I. Etude cytologique :

a) Cellules d'origine organique;

b) Cellules microbiennes (saprophytes et pathogènes).

II. Etude biochimique :
a) Les diastases ;
b) Les vitamines;
c) Les hormones;
d) Les pigments.

B. Propriftés biologiques.

I. Propriétés nutritives :

a) Valeur alimentaire (v. énergétique, biologique);

b) Digestibilité (volume, action du lab "in vivo" et "in vitro ");

c). Pouvoir laxatif ;

d) Emplois du colostrum en alimentation humaine.

II. Propriétés humorales :

a) Pouvoir infectant ;

b) Pouvoir bactéricide (P. phagocytaire, opsonique, agglutinant, lytique).

c) Pouvoir immunisant.

III. Considérations sur la finalité et la génèse du colostrum :

a) Finalité ;

b) Genèse. 


\section{CHAPITRE III. - LA COLOSTRALISATION.}

A. Pouvolr et taux d'enrichissement en holoprotétdes.

\section{Définitions.}

II. Variations :

a) Mélange d'un colostrum à du lait normal ;

b) Mélange de colostra à du lait normal :

Périodes colostrales superposées;

Périodes colostrales chevauchantes;

Périodes superposées et ehevauchantes.

B. Colostralisation des laits du commeroe.

I. Laits vendus à l'état cru.

II. Laits destinés à l'industrie laitière.

C. Importance pratique DE LA colostralisation.

CHAPITRE IV. - LA COLOSTRALISATION ET L'INDUSTRIE LAITIERE.

A. L'indUSTRIE dU LAIT DE CONSOMMATION.

I. Le transport du lait cru.

II. L'épuration du lait.

III. Homogénéisation:

a) Sédịment du lait homogénéisé ;

b) Rancidité du lait et du lait homogénéisé.

IV. Pasteurisation :

a) Montée de la crème;

b) Coagulation des holoprotéides. Conséquences ;

c) Propriétés diastasiques des laits eolostroïdes.

V. Le lait coloströ̀de à la consommation.

B. L'INDUSTRIE DES LATTS DE CONSERVE.

I. Le lait stérilisé.

II. Le lait condensé non sucré.

III. Le lait condensé sucré.

IV. Les laits en poudre.

C. LES INDUSTRIES DE LA CREMERIE ET DE LA BEURRERIE.

I. L'écrémage.

II. Composition et qualité des crèmes :

a) Constituants de la membrane des globules gras;

b) Protéines ;

c) Altération de la crème.

III. Pasteurisation des crèmes. 


\section{Barattage :}

a) Durée du barattage et pertes de matières grasses ;

b) Mousse.

V. Aspect, composition et qualités du beurre :

a) Coloration ;

b) Constantes;

c) Altérations.

D. LES INDUSTRIES DE LA FroMAGERIE ET DE LA CASÉtNERTE.

\section{Fromagerie :}

10 Les fromages de lait :

a) Maturation, épuration et écrémage du lait;

b) Coagulation du lait ;

c) Egouttage et qualité des fromages ;

d) Pasteurisation du lait en fromagerie :

2. Les fromages de lacto-sérum :

a) Le sérac ;

b) Les lacto-protéines.

II. Caséinerie.

10 Caséine-présure :

a) Caséine présure première ;

b) Caséine présure seconde ;

20 Caséine lactique et caséine aux acides.

CHAPITRE V. - RÉSUMÉS ET CONCLUSIONS.

BIBLIOGRAPHIE.

\section{INTRODUCTION}

Le colostrum (1) est le lait sécrété pendant les jours qui suivent la mise bas.

(1) Colostre ou Colostrum : du latin Colostrum, i (Pline, Servius, Martial), neutre premier lait produit après la parturition. Le mot a également une autre origine latine : Colostra, æ (Columelle, Pline), féminin - et probablement une origine grecque :

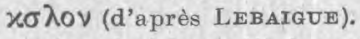

Au pluriel, on dit des colostrums, et pensons-nous, plus logiquement des colostra.

Colostral, aux : mot dérivé de colostra - qui a les caractères du colostrum, qui a rapport au colostrum.

Colostralisation : phénomène naturel ou artificiel qui tend à imprimer le caractère colostral. (Notons que le mot colostration, du latin * colostratio *, désigne la maladie des nouveau-nés due à l'action du lait.)

Colostroide : suffixe du grec, eildos $=$ forme - qui ressemble au colostrum.

Le lait colostroïde est dope un lait dont les caractères se rapprochent, de loin ou de près, de ceux du lait colostral.

Nous nous excusons d'utiliser ces derniers néologismes un peu lourds mais cependant indispensables à la clarté de l'exposé. 
Sa composition et ses propriétés ne ressemblent pas à celles du lait normal. Mais l'état colostral n'est qu'un état transitoire : au fur et à mesure que l'on s'éloigne de la parturition les différences s'atténuent et le colostrum s'identifie bientôt au véritable lait.

Pareille évolution, simple étape dans la sécrétion lactée, ne réclame qu'un temps assez court, moins bref cependant que beaucoup le pensent.

En effet, les laits colostraux dont l'aspect extérieur est déjà celui du lait courant n'ont pas encore une composition absolument identique à celle de cet aliment.

Pratiquement, il en résulte que certains d'entre eux sont livrés au commerce ou à l'industrie et se trouvent mélangés au lait normal.

Les nombreux veaux trop jeunes présentés sur les marchés d'animaux de boucherie, la fréquence des saisies de viande de veau dans les abattoirs pour les motifs d' "extrême jeunesse " ou d' "immaturité " sont de fortes présomptions indiquant que le colostrum est utilisé à d'autres fins qu'à l'alimentation des animaux.

Si le mélange de ce produit au lait normal n'avait qu'un caractère d'exception, l'intérêt du sujet serait très amoindri. Cela n'est pas. Dans notre pays, la majorité des mise bas se produisent à la même époque de l'année, le plus souvent au printemps, quelquefois en automine.

A certaines époques, on trouve donc dans les laits destinés à la consommation en nature ou à la fabrication de produits dérivés, une forte proportion de laits colostraux. Le lait normal devient un lait " colostroïde ».

Pour l'industrie laitière, les conséquences de tels mélanges, minimes à priori, sont, après examen, suffisamment importantes pour être étudiées d'une façon sérieuse.

Les méfaits des laits colostraux prennent encore plus d'intérêt, si l'on pense qu'ils s'ajoutent à ceux des laits de mammite et de rétention qui ont des origines assez analogues et ne sont pas moins négligeables puisqu'il existe des régions à mammite, des étables, des jours ou des époques à lait de rétention. C'est là un des côtés de l'altération de la composition normale des laits de mélange par les laits anormaux, que nous n'aborderons pas, mais qui mériterait un examen semblable à celui que nous allons entreprendre.

Pour saisir de façon satisfaisante les développements qui intéressent l'industrie laitière, il importe d'exposer auparavant la composition et les propriétés principales du colostrum.

A cette occasion, nous essayerons de rassembler, résumer et 
discuter les nombreux documents parus sur ce lait sans prétendre du reste ne pas en avoir omis.

Le phénomène de la "colostralisation" sera ensuite étudié dans ses origines et son importance.

Il sera dès lors plus facile de faire ressortir tout l'intérêt que l'on peut attacher à ses répercussions en industrie laitière et d'examiner les moyens d'y remédier.

Nous remercions vivement M. VUILLAUMe, professeur à l'Ecole Nationale Vétérinaire d'Alfort et M. ThieuLin, agrégé des Ecoles Nationales Vétérinaires, pour l'aide précieuse qu'ils ont bien voulu nous apporter dans l'élaboration de ce travail.

\section{GÉNÉRALITÉS}

Parmi les produits normaux élaborés par la mamelle on peut distinguer :

a) Le précolostrum ;

b) Le colostrum comprenant : le colostrum ante-partum et le colostrum post-partum ;

c) Le lait.

a) Le précolostrum est secrété pendant la première partie de la gestation au moment du repos fonctionnel de la glande mammaire. Il existe également dans les mamelles des génisses vierges.

WoODMANn et HaMmond [271] ont précisé en 1922 que jusqu'à la vingtième semaine de la gestation des vaches primipares, c'est-àdire tant que la glande mammaire n'est constituée que par des canaux galactophores dont les cellules conservent les fonctions des cellules de l'épiderme cutané, ce produit a sensiblement la composition suivante :

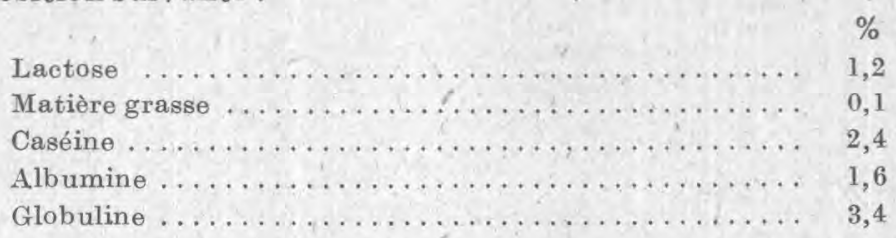

Le préeolostrum est un liquide séreux, clair, dont la quantité est relativement constante chez un animal déterminé, la sécrétion étant équilibrée par la résorption.

A partir du cinquième mois, alors que les alvéoles commencent à se développer, la sécrétion se modifie. Pour les mêmes auteurs les constituants caractéristiques du lait tendent à disparaître et 
la sécrétion, qui a la consistance du miel, contient environ $40 \%$ de constituants solides dont $35 \%$ de globuline. Cette sorte de miel est remplacée peu à peu par un liquide laiteux dont la composition tend de plus en plus vers celle du colostrum.

Depuis cette époque, Pietrke [171, 172, 173] a procédé à l'analyse du miel colostral chez les vaches primipares ou pluripares.

Les miels colostraux sont des substances blondes, souvent ambrées, onctueuses, se déshydratant rapidement pour former des paillettes de saveur fade, de réaction légèrement alcaline au tournesol.

Cette "excrétion prélactée ", ainsi que la désigne l'auteur, a la composition moyenne suivante :

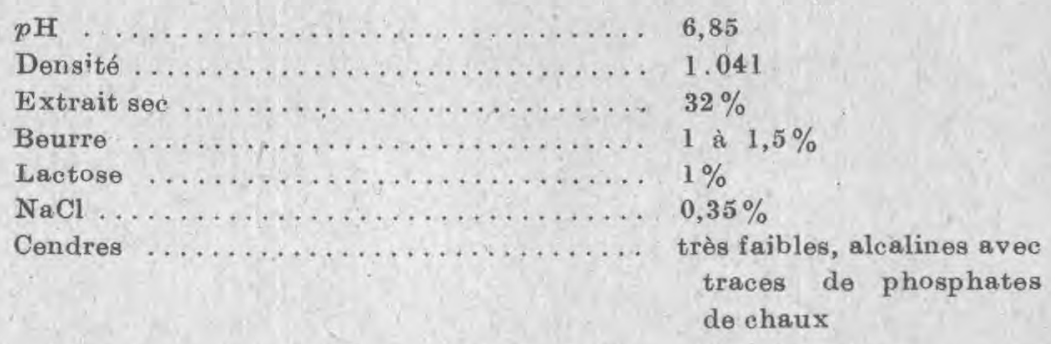

Chez les primipares, les matières grasses butyrisées apparaissent les premières, parfois vers le neuvième mois de gestation. Le sucre se montre dès le quatrième ou cinquième mois, mais son taux reste faible. La teneur assez variable en chlorures $(0,35$ à $0,43 \%$ exprimés en $\mathrm{NaCl}$ ) est plus élevée que dans le lait normal. Les phosphates calciques, encore à l'état de traces cinq à six semaines avant la parturition, augmentent dès que l'on atteint la troisième ou deuxième semaine.

Les protides constituent en poids la partie la plus importante de l'extrait sec. Pietrene soutient qu'ils sont formés par trois protéines : la globuline, l'albumine et une substance qu'il désigne sous le nom de "myxoprotéine", en quantité le plus souvent supérieure aux deux autres et à laquelle il attribue un rôle précurseur dans la formation des protéines solubles du lait.

Quant à la caséine, elle n'est présente qu'en très faibles quantités. Aux approches de la parturition, myxoprotéine et albumine diminueraient pour faire place à la caséine.

En bref, la formation du précolostrum correspond à une activité trophique de la mamelle pendant sa période de repos, activité complètement indépendante de l'activité fonctionnelle qui lui succède.

Il est certain que l'étude du précolostrum présente un réel 
intérêt. Elle peut en effet contribuer à jeter un jour sur les origines et la formation des constituants du lait ainsi que sur le développement et le fonctionnement de la mamelle.

\section{b) Le colostrum.}

Définition. Le colostrum est la sécrétion mammaire qui précède le lait. Sa composition tend vers celle du lait. Elle est fonction de l'intensité des processus de sécrétion et de résorption dont la mamelle est le siège. A compter du part et dans les conditions de l'allaitement naturel, la limite physiologique maximum de la période colostrale chez la vache semble être de vingt-deux jours.

Colostrum ante-partum. Ce produit suit le précolostrum. Il apparaît dans les semaines qui précedent la mise bas et ne diffère guère du colostrum post-partum qui lui succède, tout au moins dans les heures qui précèdent le vêlage.

Selon Mrozer et Schlag [148] on ne constate entre ces deux liquides que des différences d'ordre individuel. On gagnerait à posséder davantage de documents sur ce point.

Colostrum post-partum (1). Ce liquide est le produit sécrété par la mamelle dans les premiers jours qui suivent le part. C'est le colostrum des Anciens : premier lait qui suit la parturition.

Le présent travail est consacré à son étude et au rôle qu'il peut jouer en matière de production et d'industrie laitières.

Toutefois, il n'est pas inutile de préciser que si l'on pratique méthodiquement la traite pendant un certain temps avant le part, on peut obtenir avant celui-ci un liquide ayant l'apparence du lait, de viscosité semblable, oú la crème monte rapidement et spontané. ment, où la présure provoque une coagulation normale, comme l'ont montré Porcher et PANisset [190] chez la génisse.

Par certains côtés, ee lait ante-partum doit d'ailleurs différer du lait normal (hormones?).

Dans le travail qui va suivre, seul sera envisagé le lait que l'on recueille au moment et après le vêlage sans qu'aucune gymnastique fonctionnelle n'ait été exercée sur la mamelle pendant les jours qui précèdent la parturition : c'est-à-dire le colostrum post-partum naturel.

Pour la commodité du langage nous adopterons les termes suivants :

Le mot "colostrum " désignera simplement le colostrum postpartum naturel, liquide recueilli pendant les jours qui précèdent la sécrétion naturelle du lait normal.

(1) Dans le langage des producteurs le eolostrum est désigné sous le nom - d'amouille s. Uue vache \& amouillante n est un animal qui est sur le point ou qui vient de mettre bas. 
L'expression "premier colostrum " se rapportera à la sécrétion mammaire produite immédiatement après le vêlage, c'est-à-dire au liquide recueilli lors de la première traite effectuée aussitôt la parturition terminée (delivrance normale comprise).

\section{CHAPITRE I}

\section{ETUDE PHYSICO-CHIMIQUE DU COLOSTRUM}

Le colostrum est un liquide visqueux, collant aux doigts, de teinte allant du jaune orange au jaune clair, de goût salé et d'odeur désagréable.

Au fur et à mesure que l'on s'éloigne du part, ces caractères se modifient pour devenir peu à peu ceux du lait normal. Le produit est done d'autant plus différent du lait courant qu'il est recueilli peu de temps après la parturition.

Ces aspects, aussi bien que certaines de ses propriétés dépendent de sa constitution physico-chimique.

\section{A. CONSTITUTION PHYSICO-CHIMIQUE DU COLOSTRUM}

Tout comme le lait, le colostrum est formé d'un certain nombre de composants chimiques qui par l'état physique où ils se trouvent et par leur mode de juxtaposition, conditionnent la constitution physico-chimique du produit. C'est l'étude préalable de ces composants qui permettra de préciser cette complexion.

\section{Composition chimique}

\section{Généralités.}

Selon Duclaux "il n'y a pas un lait, mais des laits ". Dans chacun de ceux-ci, peut-on dire, "il n'y a pas un colostrum, mais des colostra".

L'état colostral n'est en effet qu'un état transitoire. La composition du colostrum n'est jamais identique à elle-même, depuis la mise en train de la sécrétion lactée jusqu'au moment où elle s'identifie à celle du lait normal. De plus cette composition dépend d'une façon spécifique, tout eomme celle du lait, de l'individualité.

Pour considérer l'ensemble chimique que formé le colostrum il est donc nécessaire de faire appel à des analyses successives et moyennes.

Au premier chef, il faut attacher un intérêt assez marqué à la composition colostrale relevée aussitôt après l'accouchement. En effet, non seulement ses extrêmes rendent compte des variations individuelles, mais encore ses moyennes sont une base de départ pour l'étude de l'évolution colostrale au cours de laquelle toutes les 
analyses n'expriment, à quelques variations près, que des transi, tions vers la composition du lait normal.

\section{$2^{\circ}$ Composition du premier colostrum.}

Les chiffres suivants sont empruntés à VAN DEN BURG [252]. Ils expriment les résultats de nombreuses analyses effectuées sur des colostra prélevés immédiatement après l'accouchement. Les compositions sont comparées à celle du lait normal (vaches hollandaises).

\begin{tabular}{|c|c|c|c|c|c|}
\hline \multirow[b]{2}{*}{ Composants \%o } & \multicolumn{4}{|c|}{ Colostrum (a) } & \multirow{2}{*}{$\begin{array}{c}\text { Lait normal } \\
\begin{array}{c}\text { Chiffres } \\
\text { moyens }\end{array}\end{array}$} \\
\hline & $\begin{array}{l}\text { Nombre } \\
\text { d'échan. } \\
\text { tillons }\end{array}$ & $\begin{array}{l}\text { Chiffres } \\
\text { infé- } \\
\text { rieurs }\end{array}$ & $\begin{array}{l}\text { Chiffres } \\
\text { moyens }\end{array}$ & $\begin{array}{l}\text { Chiffres } \\
\text { supé. } \\
\text { rieurs }\end{array}$ & \\
\hline Constituants sees & 163 & 136 & 238 & 382 & 117,5 \\
\hline Matière grasse ... & 163 & 5 & 33 & 120 & 32 \\
\hline Lactose .......... & 134 & 5 & 25 & 39 & 42 \\
\hline Matières protéiques & 155 & 66 & 170 & 266 & 32 \\
\hline Cendres $\ldots \ldots \ldots$ & 162 & 7 & 10,4 & 16,1 & 8,4 \\
\hline Poids spéeifique & 162 & 1,0366 & 1,0652 & 1,0936 & 1,0315 \\
\hline
\end{tabular}

Les données tout à fait extrêmes étant éliminées, le pourcentage de lactose dans la majorité des échantillons était compris entre 10 et $36 \%$ o.

Pour 134 échantillons la teneur en cendres fut plus élevée que $9 \%$. Pour le lait normal les données extrêmes étaient de 9,2 et $7 \%$, cette dernière concordant avec la teneur la moins élevée du colostrum.

Pour établir la moyenne des poids spécifiques, le résultat le moins élevé fut éliminé $(1,0288)$. Il correspondait à l'échantillon de colostrum le plus riche en matières grasses $(120 \%$ o).

Le premier colostrum, celui dont la composition s'éloigne le plus de celle du lait normal, a donc les caractéristiques suivantes :

Pauvreté en : eau, $826 \%$; lactose ;

Richesse en : substances sèches et notamment en : matières protéiques ; matières salines.

Les chiffres extrêmes rapportés ici donnent une idée de l'importance des variations que peut présenter la composition du

(a) Nous ne rapportons pas les chiffres extrêmes comme des données tout à fait limites. Ainsi Overmann et Sannmann [161] citent dans un tableau résumant les enseignements de vingt-einq expérimentateurs le chiffre très bas de $1,5 \%{ }^{\circ}$ de matières grasses. 
colostrum. Par l'analyse des colostra de 17 vaches lors de trois ou quatre lactations successives, le même auteur a montré que non seulement ces variations se constatent d'une vache à l'autre, mais encore pour une même vache d'une lactation à une autre.

\section{$3^{\circ}$ Evolution de la composition moyenne au cours de la période colostrale.}

Il est sans grand intérêt de rapporter les nombreux chiffres publiés à ce sujet. La diversité des moments de prélèvements, du nombre de traites, des constituants recherchés rend difficile le groupement rationnel de ces résultats. Au surplus, ils ne représentent que des états transitoires, nous l'avons dit, entre le premier colostrum et le lait normal.

Nous donnerons seulement à titre d'exemple le tableau suivant emprunté à SAvinI [219] qui donne un aperçu analytique de la composition passagère d'un colostrum quelconque :

\begin{tabular}{|c|c|c|c|c|c|c|c|c|c|}
\hline $\begin{array}{c}\text { Temps écoulé depuis } \\
\text { le vêlage }\end{array}$ & $\begin{array}{l}\text { Poids } \\
\text { spéci- } \\
\text { fique }\end{array}$ & $\begin{array}{c}\text { Ma. } \\
\text { tière } \\
\text { sèche } \\
\% \\
\% 0\end{array}$ & $\begin{array}{l}\text { Lac- } \\
\text { tose } \\
\% \text { \% }\end{array}$ & $\begin{array}{c}\mathrm{Ma}- \\
\text { tière } \\
\text { gras- } \\
\text { se } \\
\% \text { o }\end{array}$ & $\begin{array}{c}\text { Pro- } \\
\text { téines } \\
\text { tots- } \\
\text { les } \\
\% \text { o }\end{array}$ & $\begin{array}{c}\text { Ca. } \\
\text { séine } \\
\% a\end{array}$ & $\begin{array}{c}A^{1} \mathrm{bu}- \\
\text { mine } \\
\%\end{array}$ & $\begin{array}{l}\text { Glo- } \\
\text { bu- } \\
\text { line } \\
\% \text { o }\end{array}$ & $\begin{array}{l}\text { Cen- } \\
\text { dres } \\
\% 0\end{array}$ \\
\hline 0 heure & 1.068 & 228,8 & 27,4 & 23 & 122 & 48,6 & 14,5 & 53,2 & 10,3 \\
\hline 12 heures ( 1 ex jour) & I. .037 & 162,3 & 28,5 & 24,9 & 69,8 & 33,5 & 10,1 & 20,5 & 8,7 \\
\hline 24 heures ( $1^{\text {er }}$ jour) & 1.035 & 151,6 & 33,8 & 34,1 & 58,3 & 31,0 & 11,6 & 14,5 & 8,7 \\
\hline 36 heures $\left(2^{\text {e }}\right.$ jour $)$ & 1.030 & 159 & 36,3 & 47,5 & 46,5 & 27,1 & 7,8 & 6,6 & 8,2 \\
\hline 48 heures ( 2 e jour) & 1.029 & 157,4 & 36,4 & 51 & 40,2 & 26,2 & 5,2 & 5,6 & 8,2 \\
\hline 60 heures ( $3^{e}$ jour) & 1.029 & 157,5 & 38,6 & 45,5 & 40,9 & 25,6 & 5 & 4,9 & 8,2 \\
\hline 72 heures $\left(3^{e}\right.$ jour $)$ & 1.030 & 157,2 & 39,2 & 55 & 34,6 & 22,1 & 6,3 & 3,2 & 8,0 \\
\hline 84 heures ( $4^{\mathrm{e}}$ jour) & 1.030 & 156,2 & 45,8 & 54,7 & 33,6 & 21,8 & 6,1 & 2,7 & 8,0 \\
\hline 96 heures ( $4^{\mathrm{e}}$ jour) & 1.031 & 154,8 & 42,2 & 56,3 & 33,5 & 21,5 & 5,9 & 2,6 & 8,2 \\
\hline 108 heures (5e jour) & 1.031 & 160,2 & 37,8 & 53,7 & 34,8 & 24,3 & 6,2 & 2,5 & 8,6 \\
\hline 120 heures (5e jour) & 1.031 & 159,7 & 38,2 & 50,5 & 35,2 & 25,2 & 6 & 2,3 & 8,5 \\
\hline 132 heures ( $6^{\mathrm{e}}$ jour) & 1.031 & 161 & 48 & 51,5 & 30,6 & 19,7 & 5,5 & 2,1 & 8,4 \\
\hline 156 heures ( $7^{e}$ jour) & 1.031 & 165,5 & 40,1 & 51,6 & 32,2 & 22,0 & 5,6 & 2 & 8,4 \\
\hline 180 heures ( $8^{e}$ jour) & 1.031 & 162,8 & 50,1 & 49 & 32,3 & 23,4 & 5,5 & 1,9 & 8,4 \\
\hline 204 heures ( $9^{\mathrm{e}}$ jour). & 1.031 & 160,9 & 49 & 47,1 & 33,1 & 23,2 & 5,7 & 1,9 & 8,2 \\
\hline 228 heures ( $10^{\circ}$ jour) & 1.030 & 160,6 & 48,7 & 48 & 33,4 & 22,5 & 5,6 & 2 & 8,1 \\
\hline
\end{tabular}

Néanmoins, il se dégage des analyses et recherches pratiquées un certain nombre de faits intéressants à relater :

a) Les variations de la teneur en matières grasses.

Au cours d'une même période le taux de la matière grasse 
subit des variations extrêmement diverses. Il n'obéit pas aux lois générales que respectent les autres composants du colostrum :

Lois progressives de :

Diminution pour les protéines et les matières salines;

Augmentation pour l'eau et le lactose.

Ce n'est là qu'un cas particulier de cette grande règle qui veut que dans le lait la matière grasse ait des variations très amples tandis que l'extrait sec dégraissé conserve une fixité relative. Si le taux de ce dernier change au cours de la période colostrale, il ne le fait que progressivement et sans présenter de variations de caractère désordonné semblables à celles de la matière grasse.

On trouvera sans doute un jour, aussi bien pour le colostrum que pour le lait, l'origine de ces oscillations qui, probablement, sont liées aux. apports dans la mamelle, de quantités variables de substances servant à l'édification de la graisse du lait par les cellules mammaires, ou peut-être à des variations de la vie cellulaire de la mamelle sous des influences encore inconnues.

\section{b) Les variations des autres composants.}

L'examen des chiffres montré d'une façon remarquable la progression croissante ou décroissante des taux des composants autres que la matière grasse.

Ceci est si évident que Grimmer [75] a essayé d'en dégager des lois mathématiques. Malheureusement la formule obtenue ne s'applique seulement encore qu'aux taux de l'extrait sec dégraissé, de la substance azotée totale et de l'albumine du sérum. Les graphiques construits ne sont pas des droites, mais des courbes qui ont l'allure de celles traduisant des phénomènes de catalyse, selon la loi d'action des masses.

Par le seul examen des chiffres, on peut dire que dans l'ensemble l'augmentation ou la diminution des composants est très importante dans les premiers jours ( $\mathrm{I}^{\mathrm{er}}, 2^{\mathrm{e}}, 3^{\mathrm{e}}$ jour) de la période colostrale. Par la suite ces progressions sont beaucoup moins marquées en se répartissant sur un temps nettement plus long.

En voici un exemple concernant les matières protéiques emprunté à Bonn [23] (1) :

(1) Le décret du 25 mars 1924 évoque le colostrum en ces termes : s Lait provonant d'une traile opérée moins de sept jours après le part. " 


\begin{tabular}{|c|c|c|c|}
\hline \multicolumn{4}{|c|}{ 1er jour (vêlage) } \\
\hline $2^{e}$ jour & $\ldots \ldots \ldots \ldots \ldots \ldots \ldots \ldots$ & 81,7 & Chute brusque \\
\hline $3^{\mathrm{e}}$ jour & $\ldots \ldots \ldots \ldots \ldots \ldots \ldots \ldots \ldots$ & 58,4 & \\
\hline $4^{\mathrm{e}}$ jour & 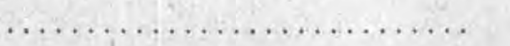 & 53,7 & \\
\hline $5^{\text {e jour }}$ & $\ldots \ldots \ldots \ldots \ldots \ldots \ldots \ldots \ldots \ldots$ & 49,3 & \\
\hline $6^{\mathrm{e}}$ jour & $\ldots \ldots \ldots \ldots \ldots \ldots \ldots \ldots \ldots$ & 49,8 & \\
\hline $7^{e}$ jour & $\ldots \ldots \ldots \ldots \ldots \ldots \ldots \ldots \ldots \ldots$ & 45,1 & \\
\hline $8^{\theta}$ jour & $\ldots \ldots \ldots \ldots \ldots \ldots$ & 42,7 & \\
\hline $9^{e}$ jour & $\ldots \ldots \ldots \ldots \ldots$ & 36,3 & Diminution progressive \\
\hline $10^{\mathrm{e}}$ jour & $\ldots \ldots \ldots \ldots \ldots \ldots$ & 33,4 & \\
\hline $11^{\mathrm{e}}$ jour & $\ldots \ldots \ldots \ldots \ldots \ldots \ldots \ldots \ldots \ldots$ & 33,5 & \\
\hline $12^{\mathrm{e}}$ jour & $\ldots \ldots \ldots \ldots \ldots$ & 32,3 & - \\
\hline $13^{e}$ jour & $\ldots \ldots \ldots \ldots \ldots \ldots \ldots \ldots$ & $3 \varkappa, 2$ & \\
\hline $14^{\mathrm{e}}$ jour & 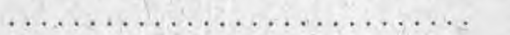 & 26,6 & \\
\hline $15^{\mathrm{e}}$ jour & $\ldots \ldots \ldots \ldots \ldots \ldots \ldots \ldots \ldots$ & 32,0 & \\
\hline
\end{tabular}

C'est aussi par l'examen des chiffres que l'on peut connaître la vitesse de variation de la composition du colostrum pour déterminer exactement le moment où le lait sécrété devient normal.

Il est certain, écrivait Porcher [183] en 1927, que plus la mise en train de la glande à la naissance sera rapide et vigoureuse, plus le balayage de la glande pleine de colostrum, balayage de sens centrifuge, sera marqué, plus les traites seront faites avec soin et poussées à fond, plus rapidement on arrivera au lait proprement dit.

Il faut noter que cette opinion fondée n'est pas en accord avec celle d'Overmann et SanManN [161] qui estiment que la vitesse de variation de composition du colostrum est indépendante de la fréquence des traites.

Quoi qu'il en soit, on est surtout frappé par ce fait que cette vitesse n'est pas constante pour tous les composants considérés ensemble.

Ce n'est pas au même instant que tous les taux des composants du colostrum atteignent ceux des constituants du lait. Chaque élément chimique ou chacun de leurs groupes à sa vitesse propre d'évolution pondérale.

C'est ainsi que le taux des matières salines atteindrait rapidement une valeur constante : vingt-quatre heures pour SнAн [220].

Dans son étude sur les variations de la teneur en protéines du lait, Azarme [5] a constaté que l'azote protéinique total diminue du début de la lactation jusqu'à la quatrième semaine.

Le taux normal du lactose serait rejoint en six à huit jours, tandis que celui des substances azotées, notamment des protéines 
solubles et coagulables ne le serait qu'après douze jours (ENGEL et Schlag [61]).

Ce sont donc les cristalloïdes qui atteignent leur taux normal les premiers.

Pour ces derniers auteurs la période colostrale serait terminée chez la vache vers le douzième jour. Sa fin peut-être fixée-d'une manière "indubitable " lorsque la teneur en protéiques est devenue normale.

Ce dernier point, semble-t-il, ne peut-être accepté sans une certaine réserve. On ne peut, en effet, fixer scientifiquement la durée maximum de la période colostrale en se basant uniquement sur le taux des composants chimiques les plus importants. Il faut attendre de posséder des précisions sur d'autres points, en particulier à l'égard des éléments constitutifs de certains composants chimiques, au sujet des diastases, des vitamines, des hormones, des cellules organiques, de la faculté de coagulation par la présure, de la consistance du coagulum ainsi que sur les possibilités d'une durée colostrale variable avec la vie physiologique de nos animaux (nombre de parturitions, lactation prolongée, etc.).

Néanmoins pour ehaque élément pris en particulier, on ne peut fixer une limite que si celle-ci traduit un changement dans l'augmentation ou la diminution. Le taux de l'élément considéré doit devenir stationnaire ou ehanger nettement le sens ou la vitesse de sa variation. Tel composant qui diminue ou augmente insensiblement depuis le vêlage jusqu'à une période avancée de la lactation ne peut ou ne pourrait servir à préciser la limite cherchée.

Si parfois la période colostrale est très brève ( 24 heures selon Siegfeld [226]), il est des cas où elle se prolonge plus longtémps que de coutume. Ce sont là des constatations d'ordre individuel en rapport sans doute avec des constitutions génotypiques différentes.

Peut-on fixer la durée physiologique maximum de cette période? Toutes considérations de diététique humaine mises à part, on expliquerait ainsi certaines variations aberrantes tout en apportant des documents à l'expert.

Critiquant les définitions accordées au colostrum, Rocharx et TAPERNOUX [208] s'expriment comme suit :

« Tout ce que l'on peut dire, e'est que le colostrum précède le lait et que sa composition après le part tend vers celle du lait qui est finalement sécrété entre le troisième et le quatorzième jour après le part. " C'est un produit variable dont la composition est fonction du temps et dont la limite est le lait. "

Pour plus de précisions, nous ajouterons à ees données générales qui s'appliquent à la majorité des colostra, que la période colostrale 
la plus longue qui ait été observée jusqu'à présent dans des conditions normales était de trois semaines (KoNING [112]).

\section{$4^{\circ}$ Etude chimique de quelques composants du colostrum.}

a) Eau et extrait sec.

La richesse en extrait sec du premier colostrum s'oppose à sa pauvreté relative en eau.

On peut suivre la progression décroissante des matières sèches au cours de la période, sur l'extrait sec dégraissé dont les fluctuations sont souvent importantes.

Dans les deux ou trois premiers jours, l'E. S. D. dépasse nettement la limite supérieure de 105 grammes fixée par Gros pour le lait. Par la suite il descend progressivement. Cette richesse du colostrum en E. S. D. l'oppose nettement aux laits de rétention proprement dits.

BARTLETT [9], qui exprime l'E. S.D. par rapport à 100 parties de lait privé de matière grasse, c'est-à-dire l'E. S. D. rectifié de Porcher, a constaté que la chute de ce poids de matière sèche se poursuit pendant six à huit semaines après le vêlage. L'E. S. D. des jeunes vaches est plus élevé que celui des vaches âgées.

b) Les lipides.

\section{๔) LES GLYCÉRIDES}

Il semble que l'on soit encore mal renseigné sur les glycérides colostraux et leurs proportions. Leur connaissance aurait cependant de l'intérêt, notamment dans l'industrie du beurre.

Comparaison avec les autres graisses organiques.

Par l'examen de ses constantes physiques et chimiques, les expérimentateurs admettent en gros que la matière grasse colostrale est intermédiaire entre celle du corps et celle du lait.

Voici quelques chiffres empruntés à divers auteurs mettant en parallèle les constantes du beurre de vache et celles du suif de bœuf avec celles relevées par Enger, Schlag et Mонв [62] d'une part, et OTT DE VRIES [160] d'autre part, sur le premier colostrum.

La démonstration serait sans doute plus probante encore si l'on s'adressait au lait, au colostrum et à la graisse d'une même vache.

Evolution des constantes de la matière grasse au cours de la période colostrale.

On peut déduire des résultats obtenus, quelques indications quant à la progression générale des indices de la matière grasse, c'est-à-dire aux transformations qu'elle subit.

Ajoutons toutefois qu'il ne s'agit que d'indications générales 


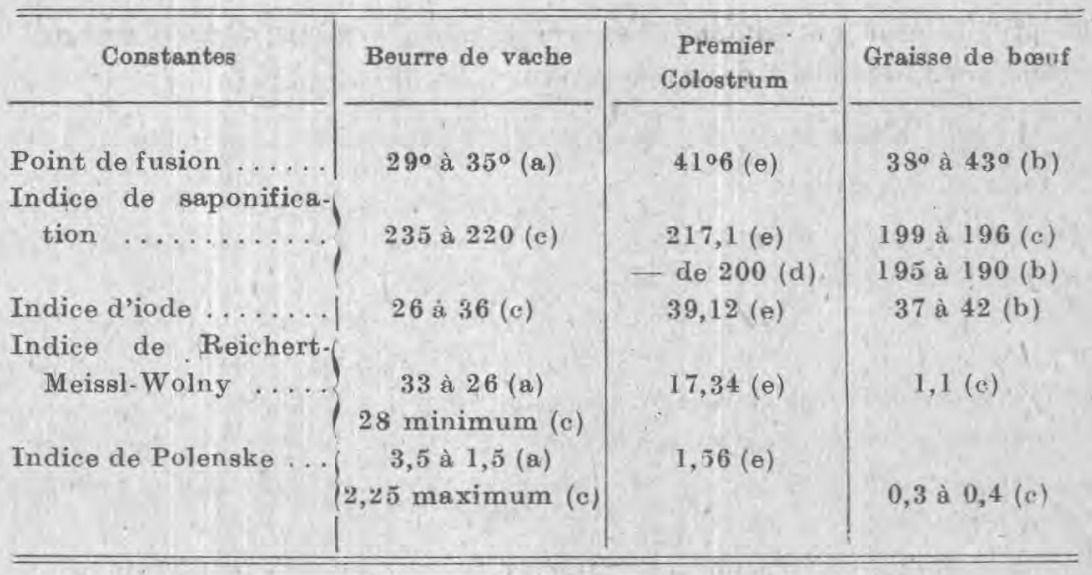

parce qu'au cours de la période, si les indices tendent à se rapprocher de ceux du beurre normal, certains ne le font qu'avec de très fortes oscillations, encore inexpliquées.

La densité augmente depuis le vêlage pour se rapprocher peu à peu de celle de la matière grasse du lait.

Les points de fusion $\left(41^{\circ} 6\right)$ et de solidification $\left(26^{\circ} 7\right)$, ainsi que l'indice de réfraction $\left(47,2\right.$ à $\left.40^{\circ}\right)$ seraient élevés lors de l'accouchement pour diminuer par la suite.

L'indice d'iode est élevé lors de la parturition et diminue dans les jours qui suivent. La matière grasse colostrale est donc plus riche en glycérides d'acide gras non saturés que celle du lait normal.

L'indice de saponification varie en sens opposé de l'indice d'iode (Enger, Schlag et Mohr [62]). C'est là une loi analogue à celle que PoLonovski et Thomas [181] ont établie pour le beurre et qui veut que les indices de Kœttstörfer, de Planchon, de Leffmann-Beam, de. Polenske, varient en sens contraire de l'indice de Hübl.

Elle montre que la régression progressive du taux des acides non saturés de poids moléculaires élevés pendant la période colostrale est compensée par l'augmentation de certains autres acides gras de poids moléculaires moins importants.

La confirmation en est apportée par le fait que les indices d'acides volatils solubles et insolubles varient également en raison inverse de l'indice d'iode, loc, cit. [62] et BoDt [21]. Ces acides augmentent au cours de la période. Au bout de six jours la matière grasse colostrale serait redevenue graisse de lait normal [62].

Cette évolution des principaux indices chimiques est confirmée par l'expérience réalisée, "in vivo " par Porcher et Muffer [189],

(a) Mangrane et Félizat [135] : (b) Prettre [174]; (c) Roohaix et Tapernoux [208]; (d) OTt de VRIEs [160] ; (e) ENger, Sohla et MoHe [62]. 
des variations allant de 11 à $93 \%$ o d'holoprotéides pour 25 à $52 \%$ o d'hétéroprotéides, soit environ deux fois plus.

ZAYKOWSKY [272] admet que le colostrum contient $130 \%$ 。 d'albumines pour $40 \%$ o de caséine, soit trois fois plus.

Dans l'exemple précédent de Monvorsin concernant une vache primipare on arrive à $155 \%$ o d'albumines pour 13 de caséine, soit environ onze à douze fois plus.

Ces différences s'expliquent sans doute, non seulement par les techniques diverses utilisées (heures des prélèvements, méthodes d'analyse...), mais aussi et surtout par des variations d'ordre physiologique (nombre de parturitions...) ou d'ordre individuel.

Il faut insister sur le fait que ces proportions se modifient très rapidement dès le $1 \mathrm{er}$, deuxième et troisième jour qui suivent le vêlage.

Ce n'est guère que dans le cas de rétention pathologique comme dans la fièvre de lait (ENGEL et Dennemark [60]) ou de rétention par absence de traite ou de succion, que ces proportions restent stables.

On sait que Poroher [182] a défini et différencié les laits albumineux et les laits caséineux, en établissant, pour 100 grammes de composés protidiques totaux, le rapport :

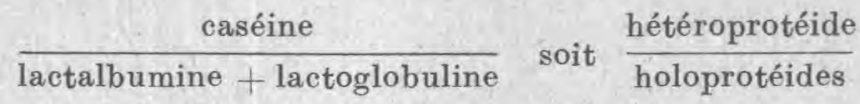

Par exemple, il donne comme type de :

Lait caséineux : le lait de vache $\ldots \ldots \ldots \ldots \ldots \ldots \frac{85}{15}=5,6$

Lait albumineux : le lait de femme ou de chienne .. $\frac{50}{50}=1$

Si nous calculons la valeur de ce rapport dans l'exemple de Monvoisin et dans eelui de SAvini, nous obtenons les chiffres ei-après.

On voit par ces nombres que le colostrum du premier âge, c'est-à-dire du premier, deuxième ou troisième jour est un lait très albumineux. A ces instants il ressemble au lait de chatte ou de chienne, puis se transforme progressivement pour devenir un lait franchement caséineux au fur et à mesure que la période colostrale s'avance.

Ce n'est là qu'une vérification de la loi énoncée par Porcher [182] du jeu inverse des molécules élaborées et des molécules non élaborées. 
COLOSTRUM DE VACHe PRIMIPARE Monvoisin (145) EN GRAMME PAR LITRE

\begin{tabular}{|c|c|c|c|c|}
\hline Date de la récolte & N total & N de la caséine & $\begin{array}{l}\mathrm{N} \text { des } \\
\text { albumines }\end{array}$ & $\mathrm{N}$ amidé \\
\hline Veille du vêlage..... & 32,10 & 4,65 & 26,20 & 1,25 \\
\hline Jour du vêlage ..... & 28,4 & 2,1 & 24,55 & 1,75 \\
\hline $2^{\mathrm{e}}$ jour $\ldots \ldots \ldots \ldots$ & 11,76 & 4,7 & 6,43 & 0,63 \\
\hline $3^{\mathrm{e}}$ jour $\ldots \ldots \ldots \ldots$ & 7,10 & 4,13 & 2,39 & 0,58 \\
\hline $4^{\mathrm{e}}$ jour $\ldots \ldots \ldots \ldots$ & 7,62 & 5,39 & 1,73 & 0,50 \\
\hline $5^{\mathrm{e}}$ jour $\ldots \ldots \ldots \ldots$ & 7,56 & 5,04 & 2,03 & 0,49 \\
\hline $6^{\mathrm{e}}$ jour $\ldots \ldots \ldots \ldots$ & 6,92 & 5,08 & 1,29 & 0,55 \\
\hline $\begin{array}{l}\text { Lait normal en gé- } \\
\text { néral } \ldots \ldots \ldots \ldots\end{array}$ & 4,7 à 5,8 & 3,7 à 4,3 & 0,9 aे 0,95 & 0,13 à 0,24 \\
\hline \multicolumn{2}{|c|}{ Soit en $\%$ de $N$ total: } & & & \\
\hline \multicolumn{2}{|c|}{ Veille du vêlage $\ldots . . . \ldots \ldots \ldots$} & 14,55 & 81,55 & 3,90 \\
\hline \multicolumn{2}{|c|}{ Jour du vêlage $\ldots \ldots \ldots \ldots \ldots$} & 7,40 & 86,45 & 6,15 \\
\hline \multicolumn{2}{|l|}{$2^{\mathrm{e}}$ jour ....... } & 39,95 & 54,67 & 5,38 \\
\hline \multicolumn{2}{|l|}{$3^{e}$ jour } & 58,1 & 33,7 & 8,2 \\
\hline \multicolumn{2}{|l|}{$4^{\mathrm{e}}$ jour. } & 70,7 & 22,7 & 6,6 \\
\hline \multicolumn{2}{|l|}{$5^{\mathrm{e}}$ jour. } & 66,6 & 27 & 6,4 \\
\hline \multicolumn{2}{|l|}{$6^{\mathrm{e}}$ jour.... } & 73,4 & 18,65 & 7,95 \\
\hline \multicolumn{2}{|l|}{ Lait normal ....... } & 77 à 81 & $\mid 15,7$ à 17,15 & 2,5 à 4,7 \\
\hline \multicolumn{2}{|l|}{ Soit en substances } & & \multirow{3}{*}{$\begin{array}{c}N \times 6,34(a) \\
166,00\end{array}$} & \multirow{3}{*}{$\begin{array}{c}N \times 9,35 \\
11,68\end{array}$} \\
\hline azotées : & \multirow{2}{*}{$\begin{array}{c}\mathrm{N} \times 6,557 \\
210,4\end{array}$} & $\mathrm{~N} \times 6,39$ & & \\
\hline Veille du vêlage .... & & 29,71 & & \\
\hline Jour du vêlage ..... & 186,31 & 13,41 & 155,64 & 16,36 \\
\hline $2^{\mathrm{e}}$ jour $\ldots \ldots$ & 77,11 & 30,03 & 40,10 & $\tilde{5}, 89$ \\
\hline $3^{e}$ jour $\ldots \ldots$ & 46,55 & 25,89 & 15,15 & 5,42 \\
\hline $4^{e}$ jour .......... & 49,96 & 34,44 & 10,90 & 4,67 \\
\hline $5^{\mathrm{e}}$ jour $\ldots \ldots \ldots \ldots$ & 49,57 & 32,20 & 12,87 & \\
\hline $6^{\mathrm{e}}$ jour..... & 45,37 & 32,46 & 8,18 & $\begin{array}{l}4,30 \\
5,14\end{array}$ \\
\hline Lait normal .. & 30,8 à 38,03 & 23,64 à 27,47 & 5,7 à 6,02 & 1,21 à 2,24 \\
\hline
\end{tabular}

portion d'albumines d'environ deux à quatre fois supérieure à celle de la caséine.

Cette proportion devient plus variable avec les recherches récentes.

Dans l'exemple donné par SAvinI [219] au début de ce travail, on trouve $67,7 \%$ o d'albumines pour $48,6 \%$ o de caséine soit 1,5 fois plus.

Les chiffres d'N établis par Crowther et RaIstrick [43] mentionnés un peu plus loin, traduits en composés azotés, indiquent

(a) Coefficient utilisé par l'auteur. Très souvent on emploie celui de 6,25 . 
en 1930. La résorption de la matière grasse injectée dans la mamelle sèche se traduit non seulement par un indice d'iode plus élevé et un indice de saponification plus faible, mais encore par une diminution importante des acides volatils solubles.

Lors de rétention lactée, la matière grasse doit disparâttre de la mamelle en suivant à rebours un processus chimique analogue à celui qu'elle parcourt lors de la sécrétion lactée.

\section{ß) LES PHOSPHOAMINOLIPIDES}

Ces corps (lécithides et céphalides) présents dans le lait normal y sont en faible quantité $(0,3 \%$ o) (LobStein et Flatter [129]).

Ils seraient plus abondants dans le colostrum que dans ce dernier. C'est ce qu'affirmait déjà Wood [269] en 1905.

La teneur du colostrum en lécithines augmente jusqu'au vêlage, mais, précise Horall [90] elle diminue ensuite assez vite pour devenir constante à partir du quatrième jour.

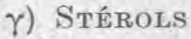

On sait que le lait normal contient une petite quantité de cholestérol $(0,10$ à $0,175 \% \mathrm{o})$. Il en existe davantage dans le colostrum, mais cette proportion diminue rapidement et, au bout de quarante-huit heures, elle est la même qu'après trọis ou sept mois de lactation, Richard, Shope et Gowen [202].

Le cholestérol débarrassé des traces de provitamine D (ergostérol) qu'il contient, peut acquérir par chauffage des propriétés antirachitiques. Sans doute est-il à l'origine du pouvoir antirachitique du lait et du colostrum que nous étudierons plus loin.

\section{c) Les composés azotés.}

Tout comme le lait, le colostrum contient des composés azotés protidiques (caséine, lactalbumine, lactoglobuline) et des composés azotés non protidiques (urée, etc.).

Avant de les étudier, donnons un exemple de leurs proportions qui sont très différentes de celles qu'ils ont dans le lait.

\section{๔) Composés azotés protidiques}

La pauvreté relative du premier eolostrum en hétéroprotéides (caséine) et sa richesse en holoprotéides (lactalbumine + lactoglobuline) sont bien connues.

Dans les recherches déjà anciennes (Koning, Fleischmann, Engling, Hansen et Schrodt, Sebelien, Thiemann, Simon) (1), on peut constater par le calcul que les auteurs indiquent une pro-

(1) D'après Brourge [18]. 


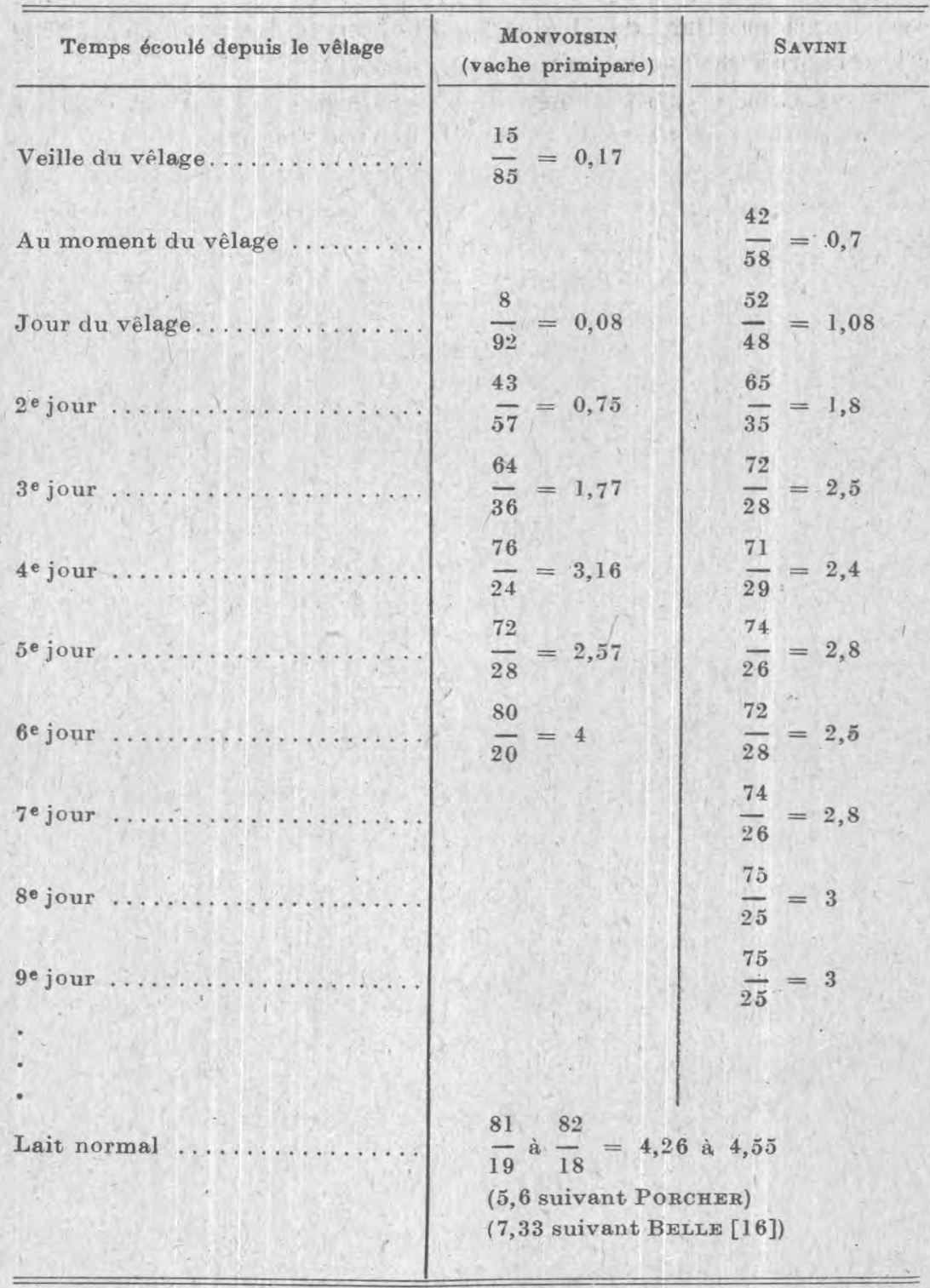

La caséine obtenue à partir du colostrum par GRoH et ALTIN [77] est soluble dans l'alcool à $70 \%$ légèrement acidifié. Elle ressemble. rait en cela à l'albumine obtenue en 1918 par OsBoRNE et WAKEMANN [156] qui ont retiré de la caséine du lait par action de l'alcool chaud, une petite quantité $(5 \%$ ) d'un protide exempt de phosphore. Elle en diffère cependant puisque sa composition est celle de la caséine. 
Si l'origine chimique de la caséine du lait est encore imparfaitement connue, l'étude de la caséine colostrale contribuera peut-être à éclairer la question.

La lactalbumine est plus abondante que la lactoglobuline dans le lait normal :

Lactalbủmine : 4 à $5 \%$ (Porcher [182]) ; $4 \%$ (DAvies [47]) ; $5,6 \%$ (autres auteurs) ;

Lactoglobuline : $0,5 \%$ (Porcher [182], BELL [13]); 1,8\%o (DAvies [47]).

Dans le colostrum, si ces deux composés sont en quantités plus fortes, c'est la lactoglobuline qui occupe la place la plus importante. Les analyses anciennes accusaient déjà cette prépondérance. En voici un exemple :

\begin{tabular}{|c|c|c|}
\hline & Albumine $\%$ & Globuline \%o \\
\hline SEBELIEN (loc. cit.) & 15,9 à 20 & 6,3 à 102,7 \\
\hline Thinmann (loc. cit.) & $0 \quad 2,5$ & $23,5 \quad 175,1$ \\
\hline & & \\
\hline
\end{tabular}

Le tableau ci-contre indique les chiffres d'azote obtenus par CRowther et Ratstrick [43] en 1916 sur huit premiers colostra :

\begin{tabular}{c|c|c|c|c}
\hline \hline N Total & N Caséine & N Albumine & N Globuline & N non protéiné \\
\cline { 3 - 5 } 24 & 7,5 & 1,4 & 13,2 & 1,9 \\
20,1 & 6,8 & 1,7 & 10,2 & 1,4 \\
14,4 & 5,9 & 1,4 & 5,9 & 1,2 \\
9,7 & 1,1 & 1,1 & 3,1 & 0,4 \\
7,6 & 4,6 & 0,7 & 2 & 0,3 \\
7,5 & 4,6 & 0,6 & 2 & 0,3 \\
6,9 & 4,2 & 0,6 & 1,8 & 0,3 \\
6,5 & 4,6 & 0,5 & 1,2 & 0,2 \\
\hline \hline
\end{tabular}

Traduits en composés azotés à l'aide du coefficient 6,34 , ces chiffres montrent des variations allant de : 3 à $9 \%$ pour l'albumine et 7 à $83 \%$ o pour la globuline.

En 1923, WooDMANN [270] a trouvé de 60 à $120 \%$ o de globuline après la mise bas. Ce taux élevé diminue rapidement dès que l'activité mammaire s'accroit nettement (deuxième phase de la période).

Si tous ces chiffres montrent encore des variations étendues qui marchent de pair avec celles des holoprotéides totaux, on peut 
dire toutefois que lors de la mise bas, les holoprotéides sont très abondants dans le colostrum avec prédominance de la globuline, tandis qu'à la fin de la période colostrale ils sont très réduits avec prépondérance de l'albumine. En d'autres termes, le colostrum de vache est plus un lait globulineux qu'un lait albumineux.

Spécificité des protéines du colostrum.

De nombreuses recherches ont été effectuées sur la spécificité des sérums anti- préparés avec les protéines du lait ou du colostrum.

BAUER et ENGEL [12] ont constaté que les trois matières protéiques du colostrum se comportent absolument comme celles du lait. Pour ces auteurs globuline et albumine seraient identiques dans les trois milieux : sang, colostrum, lait.

Cependant Crowther et RaIstrick [43], puis plus tard Woodmann [270] ont montré que si les globulines du sérum sanguin, du lait et du colostrum sont identiques, l'albumine du sérum se différenciait de celle du colostrum et du lait. Ces constatations jettent un jour sur l'origine des protéides du lait en général.

Comme le suppose Rimington [205], les protéines du sang fournissent le matériel qui sert à la synthèse de la caséine par la cellule mammaire. La facile perméabilité de cette cellule à la sérum-globuline, qui résulte de l'identité de cette protéine dans les trois milieux sang, colostrum, lait, plaide en faveur de cette hypothèse, avec cette réserve que les proportions contenues dans le colostrum et dans le lait sont très différentes.

Cette opinion sur le rôle précurseur de la globuline est appuyée par certains faits expérimentaux :

10 La globuline du sang, phosphorylée, coagule par la présure (NeUBERg et OERTELL) [150];

$2^{\circ} \mathrm{Au}$ cours de la rétention lactée, il se formerait de la globuline aux dépens de la caséine sous l'action des ferments solubles sécrétés par les leucocytes (MUFFET) [149].

A ce sujet, Porcher [183] s'exprimait ainsi :

"Comme le colostrum est un produit de rétention, on peut se demander si la globuline ne dérive pas de la caséine sous l'intervention des diastases protéolytiques sécrétées par les phagocytes, toujours abondants en l'espèce. "

Nous avons vu que eette opinion, qui n'est qu'une hypothèse de travail, n'est pas partagée par Pietrre qui place la myxoprotéine à l'origine des protéines du lait et du colostrum.

Quoi qu'il en soit, le caractère spécifique du colostrum, comme celui du lait, relève surtout de son albumine et de sa globuline, beaucoup moins que de sa caséine qui paraît être un antigène banal. 


\section{ß) Composés azotés noN pRotidiques}

Par l'examen des chiffres rapportés plus haut, on voit que le colostrum contient, comme les laits de rétention en général, une assez forte proportion de composés azotés non protidiques.

Le taux de ces substances suit aussi la loi de la diminution progressive au cours de la période. Nous n'envisagerons que deux d'entre eux :

L'urée. - En 1922, Мовімото [144] a trouvé $0 \mathrm{gr} .75$ d'urée dans le colostrum après la mise bas soit plus de la moitié des composés azotés non protidiques. Cette quantité est nettement supérieure à celle que l'on décèle normalement dans le lait $(0 \mathrm{gr}$. 20 à $0 \mathrm{gr}$. 30).

L'ammoniaque. - Alors que le lait frais et aseptique ne contient que $0 \mathrm{mgr}$. 2 à 1 milligramme d'ammoniaque par litre, le colostrum peut en renfermer de 15 à 20 milligrammes par litre (PoLonovski et BoulaNG ER [179, 180]).

\section{d) Les glucides.}

๙) Le Lactose. - La teneur du premier colostrum en lactose est faible. Elle augmente peu à peu par la suite. Ce faible taux est compensé suivant la loi de Porcher, par une teneur élevée en chlorures sur laquelle nous reviendrons plus loin. Dans deux cas, qui apparaissent d'ailleurs comme exceptionnels, il n'a pas été trouvé de lactose dans le premier colostrum (Crusius [44], OvERMann et SANMANN [158]).

$\beta$ ) Les autres sucres. - Polonovski [178] a montré que le colostrum de femme est plus riche en gynolactose que le lait normal.

Witnah et CAULFIELD [265] ont signalé dans le lait la présence d'un sucre ressemblant au glucose. La recherche des sucres rares dans le colostrum de vache apportera peut-être des éclaircissements sur la présence, la formation ou les transformations des sucres dans le lait.

Déjà Polonovski a entrepris des recherches sur ce sujet [177]. Le rapport des coefficients réducteurs après et avant hydrolyse de l'holoside ( 1,40 pour le lactose) a été supérieur à 1,47 sur un défécat silico-tungstique de colostrum frâ̂chement trait.

L'indice chromique (méthode d'oxydation sulfochromique ménagée) correspondait également à une teneur en glucides totaux supérieure de $15 \%$ au seul lactose calculé d'après le dosage suivant la méthode de Bertrand.

De plus, et c'est là le point le plus curieux, le pouvoir rotatoire observé était bien plus dextrogyre que ne le comportait la quantité de lactose dosé.

Ces constantes optiques et réductrices tendaient d'ailleurs 
vers celles du lactose ordinaire lorsque le colostrum était abandonné à lui-même au laboratoire. C'est pourquoi les recherches ultérieures furent pratiquées sur 120 litres de colostrum fixé par la chaleur ou l'alcool immédiatement après la traite.

Le dérivé glucidique obtenu par cristallisations successives de sa solution méthylique a donné un rapport $=1,84$ et un pouvoir rotatoire presque double du pouvoir rotatoire théorique calculé pour le lactose d'après le pouvoir réducteur.

Il s'agit probablement d'un ester phosphorique d'ose que l'auteur cherche à obtenir à l'état pur.

A l'appui de cette opinion, notons, d'après Overmann et Sanmann [158] que Ies différences entre les résultats du dosage du lactose par la méthode optique et "par différence" sont plus importantes au début de la période colostrale qu'à la fin.

e) Les matières salines.

D'une façon générale les chercheurs sont d'accord pour reconnaître que le colostrum est riche en matières salines. C'est ce qu'exprime le taux des cendres qui ne sont que le résidu de la calcination de ces matières. Un tel caractère oppose le colostrum aux autres laits de rétention.

La quantité de cendres retirée du premier colostrum varie d'une façon plus étendue que celle extraite du lait normal. Si, dans ses extrêmes inférieurs, elle ne descend guère au-dessous du quantum le plus bas trouvé pour le lait courant $(7 \%$ o), elle atteint par contre des chiffres très élevés dans ses limites supérieures. C'est ainsi que BAUER [11] indique le chiffre très haut de $19,7 \%{ }^{\circ}$.

Il semble que l'on soit encore assez mal renseigné sur les proportions des différents sels du colostrum, sur leur état et leur vitesse de variation.

\section{๔) Les éléments de Base}

Chlore et sodium. - VAN DEN BURG [252] dosant les chlorures sur 127 échantillons donne un taux moyen de 1 gr. $655 \%{ }^{\circ}$ (variations extrêmes $0 \mathrm{gr}$. 538 à $4 \mathrm{gr}$. $605 \%$ - vaches hollandaises).

Le colostrum a done bien une teneur légèrement plus élevée en chlorures puisque le lait normal correspondant n'en contient que $1 \mathrm{gr} .11 \%$ o d'après le même auteur $(1,8$ à $2 \%$ o dans le lait courant, d'après PoRcher). Ceci explique la saveur salée du colostrum.

La teneur en chlorures diminue pendant les quelques jours qui suivent le début de la lactation pour rester ensuite constante pendant environ $60 \%$ de la durée de cette lactation (ScHARP et Strubble [221]).

Si une partie du chlore est fixée sur le potassium, la plus grande 
partie l'est sur le sodium. VIVARIo et StaInIER [255] indiquent une décroissance du $\mathrm{NaCl}$ allant de 2 gr. 9 au premier jour à 1 gr. 86 le huitième jour (voir phosphore).

Phosphore. - Le colostrum est riche en phosphore (TRUnz [245]). Pour Stainier [233], c'est là un caractère qui l'oppose aux laits de rétention en général dont le phosphore minéral suit l'abaissement du lactose comme nous le verrons plus loin.

VIVario et Stainier donnent les chiffres suivants :

\begin{tabular}{|c|c|c|c|c|c|}
\hline$\%$ & $\mathrm{NaCl}$ & $\mathrm{Cl}$ & $\begin{array}{l}\text { P Total } \\
\text { en } \mathrm{P}_{2} \mathrm{O}_{5}\end{array}$ & $\begin{array}{l}\mathrm{P} \text { Minéral } \\
\text { en } \mathrm{P}_{2} \mathrm{O}_{5}\end{array}$ & $\begin{array}{c}\mathrm{P} \text { soluble } \\
\text { en } \mathrm{P}_{2} \mathrm{O}_{5}\end{array}$ \\
\hline 1er jour ....... & 2,9 & 1,74 & 4,32 & 3,37 & 2,35 \\
\hline 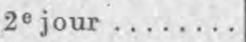 & 2,57 & 1,54 & 3,94 & 3,24 & 2,35 \\
\hline $3^{e}$ jour ... & 2,53 & 1,52 & 3,35 & 2,73 & 2,19 \\
\hline $4^{\mathrm{e}}$ jour ... & 2,25 & 1,35 & 2,6 & 2,13 & 1,72 \\
\hline $5^{e}$ jour... & 2,09 & 1,26 & 2,54 & 2,03 & 1,64 \\
\hline $6^{e}$ jour ....... & 2 & 1,2 & 2,54 & 1,94 & 1,50 \\
\hline $7^{\mathrm{e}}$ jour .... & 1,99 & 1,19 & 2,54 & 2,03 & 1,48 \\
\hline se jour....... & 1,86 & 1,12 & 2,51 & 1,94 & 1,29 \\
\hline
\end{tabular}

Le phosphore minéral comprend les phosphates alcalins et les phosphates alcalino-terreux, tous solubles dans l'acide trichloracétique.

Le phosphore solpble est celui qui est soluble dans le lait et passe dans le sérum obtenu après action de la présure. Il est constitué par des phosphates alcalins.

Les chiffres ci-dessus indiquent qu'à partir du neuvième jour, les taux. du phosphore et du chlorure de sodium ont atteint leur taux normal.

Cependant, OLson [154] soutient que la teneur en phosphore demande six semaines pour devenir normale.

$$
*^{*} *
$$

Pour se rendre compte de la répartition grossière des phosphates, il faut tout d'abord se reporter au travail de PORCHER et CHEVALLIER [188] sur la répartition des matières salines dans le lait. Dans les cendres de celui-ci on trouve en moyenne: 74 à $76 \%$ du phosphore total, sous forme minérale, répartis comme suit :

$\mathrm{P}_{2} \mathrm{O}_{5}$ en solution : 0 gr. 9 à 0 gr. 95 , soit 57 à $58 \%$ du $\mathrm{P}$ minéral ; $\mathrm{P}_{2} \mathrm{O}_{5}$ en suspension : $0 \mathrm{gr}$. 65 à $0 \mathrm{gr}$. 7 , soit 42 à $43 \%$ du $\mathrm{P}$ minéral.

Si nous effectuons les mêmes calculs en partant des chiffres ci-dessus de VIVARIo et StaINIER, nous obtenons pour un colostrum 
du premier jour : $78 \%$ du phosphore total sous forme minérale répartis en :

$\mathrm{P}_{2} \mathrm{O}_{5}$ en solution : $2 \mathrm{gr}$. 35 , soit $60 \%$ du $\mathrm{P}$ minéral ;

$\mathrm{P}_{2} \mathrm{O}_{5}$ en suspension : 1 gr. 02 (chiffre calculé : $3,37-2,35$ ), soit $30 \%$ du P minéral.

On en déduit que le colostrum contiendrait proportionnellement plus de phosphates solubles que le lait et moins de phosphates colloïdaux.

Pour Porcher, le phosphore est comme le lactose un élément élaboré et il varie dans le lait en sens inverse du chlore. Ceci s'exprime dans le rapport $\frac{\mathrm{P}}{\mathrm{Cl}}$ dont la valeur diminue dès que le chlore augmente.

Ainsi, d'après VIVARIo et StaINIER, cette valeur diminue dans les laits de fin de lactation et certains laits anormaux :

\begin{tabular}{|c|c|c|c|c|c|}
\hline & & $\frac{\text { minéral }}{\mathrm{Cl}}$ & $\frac{\mathrm{P} \text { so }}{\mathrm{C}}$ & $\frac{\text { oluble }}{\text { cl }}$ & $\frac{\mathrm{P}_{2} \mathrm{O}_{5} \text { total }}{\mathrm{Cl}}$ \\
\hline Laits anormaux . & 0,31 & à 0,036 & 0,036 & à 0,12 & 3,2 à 0,16 \\
\hline Laits de fin de lactation ...... & 1,16 & à 0,42 & 0,32 & à 0,14 & 1,5 à 0,62 \\
\hline
\end{tabular}

Or, le colostrum, comme d'ailleurs les laits de fin de lactation des vaches en gestation, fait exception à cette règle parce que son phosphore varie dans le même sens que son chlore.

En conséquence, la valeur du rapport s'élève :

\begin{tabular}{|c|c|c|}
\hline P minéral & P soluble & $\mathrm{P}_{2} \mathrm{O}_{5}$ total \\
\hline $\mathrm{Cl}$ & $\mathrm{Cl}$ & $\mathrm{Cl}$ \\
\hline 1,93 . & 1,93 & 2,48 \\
\hline 2,1 & 1,52 & 2,56 \\
\hline 1,8 & 1,4 . & 2,2 \\
\hline 1,58 & 1,27 & 1,92 \\
\hline 1,6 & 1,3 & 2 \\
\hline 1,6 & 1,25 & 2,1 \\
\hline 1,7 & 1,2 & 2,12 \\
\hline 1,7 & 1,15 & 2,12 \\
\hline 1,75 & 1,12 & 2,21 \\
\hline 1,84 & 1,14 & 2,3 \\
\hline 1,7 & 1,06 & 2,15 \\
\hline
\end{tabular}


Voici les nombres limites trouvés pour les différents rapports :

$$
\begin{aligned}
& \frac{\mathrm{P} \text { total }}{\mathrm{NaCl}}=1,53 \text { à } 1,21 \\
& \frac{\mathrm{P} \text { minéral }}{\mathrm{NaCl}}=1,25 \text { à } 0,97 \\
& \frac{\mathrm{P} \text { soluble }}{\mathrm{NaCl}}=0,91 \text { à } 0,69
\end{aligned}
$$

$$
\begin{aligned}
& \frac{\mathrm{P} \text { total }}{\mathrm{Cl}}=2,56 \text { à } 1,92 \\
& \frac{\mathrm{P} \text { minéral }}{\mathrm{Cl}}=2,1 \text { à } 1,6 \\
& \frac{\mathrm{P} \text { soluble }}{\mathrm{Cl}}=1,52 \text { à } 1,15
\end{aligned}
$$

Ainsi les laits de fin de lactation des vaches en gestation annonceraient le lait de colostrum où la quantité de phosphates est fort supérieure à la normale. Il semble, disent les auteurs, que l'on doive admettre une influence hormonique commandant ce phénomène. La finalité de cette constatation est évidente. Les besoins du jeune légitiment cette augmentation des phosphates.

Calcium, potassium, magnésium. - Ces trois éléments existent dans le colostrum comme dans le lait. Comme pour le phosphore la teneur en calcium est élevée au début de la lactation. Pour OLson [154] elle devient moindre au bout de six à huit semaines. La teneur en magnésium serait également élevée (Trunz [245], GarRetw et Overmann [71]). Elle diminuerait comme celle du calcium pour atteindre un nivean assez constant dès que le lait redevient normal.

Cependant Enger et Schlag [61] estiment que les teneurs en chaux et en magnésie varient extraordinairement.

Pour Garretit et Overmann [71], le taux du potassium qui est plutôt bas à la parturition remonte régulièrement pour se fixer à un niveau constant dès que le lait redevient normal. C'est ce qui pensait déjà Trunz, qui indiquait en 1904, que ce faible taux montait nettement pendant deux semaines pour n'atteindre son maximum qu'au deuxième mois de la lactation.

D'après Nоттвонм [153], comparativement au lait de fin de lactation, le colostrum est riche en potasse. Le rapport $\frac{\mathrm{K}_{2} \mathrm{O}}{\mathrm{Na}_{2} \mathrm{O}}$ ou indice d'aleali, y est supérieur à 2 , tandis qu'en fin de lactation, le sodium l'emporte souvent sur le potassium. Dans le lait normal, ce rapport varie de 2 à 10.

Acide citrique. - A l'inverse de ce que l'on avait avancé, JERLOR [96] soutient que l'acide citrique n'est présent dans le lait que le deuxième jour après la mise bas.

Cette constatation vérifie la loi qui fait varier parallèlement le taux des citrates et celui du lactose. Elle pose la question de la 
répartition dans le premier colostrum des bases (Ca-Mg-K) qui salifient normalement l'acide citrique.

Soufre. - Cet élément serait plus abondant au début de la lactation que dans le lait normal (BuRuiana et Nicol $\mathbb{E}$ [32]).

$$
\text { *** }
$$

Cet aperçu sur les matières minérales les plus importantes montre que le colostrum se rapproche par ce côté encore plus de la composition des hématies que le lait normal, tout en. s'éloignant davantage de celle du sérum sanguin.

\section{ß) Les oligoéléments MinérauX}

Fer. - On sait que le lait de vache contient 0 mgr. 5 à 1 gramme de fer par litre. La teneur du colostrum en fer est toujours légèrement supérieure à cette dernière (de $1 \mathrm{mgr}$. 10 à $1 \mathrm{mgr}$. 30 par litre) (RABOTTI [195]).

Cuivre. - La teneur du colostrum en cuivre est plus élevée que celle du lait normal.

Dans celui-ci Lesné, Zizine et Briskas [126], utilisant la méthode McFarlan, quelque peu modifiée, trouvent $0 \mathrm{mgr}$. 09 à $0 \mathrm{mgr} .5$ par litre suivant la saison.

Pour le colostrum Brock et Woolf [28], qui ont dosé le cuivre par une méthode micrométrique, indiquent le chiffre de 0,16 p. p. m. (1), alors que le lait normal ne contiendrait que 0,028 p. p. m. Ces chiffres sont moins élevés que ceux indiqués par d'autres auteurs.

Zinc. - Le colostrum renferme plus de zinc que le lait normal (Sato et Murata [216]), On y trouve $13 \mathrm{mgr} .57$ par litre immédiatement après la mise bas. Ce chiffre baisse au cours de la première dizaine de jours, Au deuxième et troisième mois il passerait à 2 mgr. 35- $2 \mathrm{mgr} .12$ pour se relever en fin de lactation (4 mgr. 58).

Pour Brock et Woolf [28], la teneur en zine serait de 5,8 p. p. m dans le colostrum et 3-4 p. p. m. dans le lait.

Manganèse. - Le lait colostral contiendrait beaucoup plus de manganèse que le lait normal. Voici les teneurs indiquées par Sato et Murata [217] (méthode colorimétrique).

$$
\begin{array}{lllc} 
& \multicolumn{3}{c}{\begin{array}{c}
\text { Chiffres extrêmes } \\
\text { mgr } \% \text { ce }
\end{array}} \\
\text { Lait normal de vache } \ldots \ldots \ldots \ldots \ldots & 0,026 & 0,048 \\
\text { Lait colostral de vache } \ldots \ldots \ldots \ldots & 0,045 & 0,210
\end{array}
$$

Les moyennes calculées par ces auteurs sont de : $0 \mathrm{mgr}$. 16 (1) p. p. m. = parties par million. 
immédiatement après la parturition, 0 mgr. 06 le quatrième jour et 0 mgr. 03 pour les laits prélevés plus tard.

$\mathrm{Au}$ sujet du manganèse il faut signaler que si ZBINDEN [273] a pu déceler le métal dans le lait de vache par la méthode spectro. graphique, Blumberg et Rask [20] et OREA [155] n'ont pas réussi à le mettre en évidence par la même méthode. Il serait donc intéressant d'appliquer cette technique au colostrum pour obtenir des précisions en la matière.

Pour Brock et Woolf [28], la teneur en manganèse serait de 0,007 p. p. m, dans le colostrum et de 0,01 à 0,007 dans le lait (méthode micrométrique), ce qui s'oppose aux constatations de Sato et Murata.

Iode. - Comparativement au lait, le colostrum contient beaucoup d'iode [Maurer et Dierz [139], Mietke et Courth [141], Fret [64]).

Après la mise bas il contiendrait $36 \gamma$ pour $100 \mathrm{~cm}^{3}$, soit dix fois plus que le lait normal. Huit heures après il n'en renfermerait plus que 20 , puis 10 après douze heures, 5 après vingt-quatre heures et finalement 3 (MAURER et Dierz [139]).

Ceci est un peu en contradiction avec les recherches de KIEFFERLe, KettNer, ZelLer et Hanusoh [106] montrant qu'immédiatement après le part, la teneur en iode du colostrum est normale. Après dix-huit heures elle augmente brusquement de 20 à $272 \gamma$ par litre, puis elle baisse rapidement, de sorte que, trente heures anrès le part, cette teneur est redevenue normale.

L'iode colostral aurait pour rôle d'exciter la sécrétion de certaines hormones (h. de la glande thyroïde) ainsi que l'ont montré KraUs et Monroe [116] chez le rat.

Silicium. - Ketrmann [104] a signalé qu'un kilogramme de lait contient en moyenne 1 mgr. 6 de $\mathrm{SiO}_{2}$, soit $0,021 \%$ des cendres. Ce taux varie d'ailleurs avec celui de la silice dans la ration.

Au début de la période colostrale, la teneur en silice est supérieure à ce taux moyen et égale 2 mgr. 4 par kilogramme.

$$
\text { ** * }
$$

Il est difficile de présenter les unes à côté des autres les compositions chimiques du plasma sanguin, du colostrum écrémé et du lait écrémé, parce que le plus souvent les chiffres indiqués se rapportent seulement à un ou quelques éléments chimiques dosés chez des animaux différents ou par des méthodes diverses.

Le colostrum apparaît cependant comme un liquide intermédiaire entre le sang et le lait : 
Soit parce qu'il renferme des composés qui caractérisent séparément ces deux liquides organiques;

Soit parce que les taux des constituants communs au sang et au lait, que l'on y découvre, sont intermédiaires entre ceux que ces mêmes constituants possèdent dans les deux liquides.

\section{Constitution physique}

Les composants chimiques du colostrum se trouvent dans des états physiques analogues à ceux des composants du lait. On peut donc dire que "le colostrum est une émulsion de matière grasse dans une solution colloïdale, dont le liquide intermicellaire est une solution complexe. "

Cependant ces trois états diffèrent de ceux du lait par le taux des éléments qui les caractérisent. Il faut insister sur ce fait, particulièrement net dans le premier colostrum et qui a pour conséquence d'imprimer au colostrum les propriétés physico-chimiques qui lui sont propres.

10 La solution vraie (lactose. sels minéraux solubles, azote non protéique).

Comme pour le lait il faut lui reconnaître deux aspects :

a) La solution ionique formée par les électrolytes. On y remarque l'absence de l'acide citrique, élément élaboré et l'abondance des phosphates solubles (c'est-à-dire des phosphates alcalins) et des chlorures.

On sait que Porcher [182] fait des phosphates alcalins les facteurs principaux de l'acidité actuelle du lait. Il en est sans doute de même dans le colostrum où le taux élevé des phosphates solubles marche de pair avec une acidité actuelle élevée $(p H=6,22)$.

La solution vraie du colostrum, riche en sels solubles, possède done une forte dissociation électrolytique.

Il faut cependant signaler que les déplacements salins ne semblent pas y être réalisés suivant les règles édictées par PORCHER [182] pour les laits anormaux ou pathologiques.

A l'augmentation des phosphates solubles ne correspond pas une augmentation parallèle de l'acide citrique et une baisse des chlorures.

A l'augmentation du taux des chlorures ne correspond pas un développement de l'alcalinité, e'est-à-dire une marche du $p H$ vers 7 ou au delà. Il est vrai que ce développement est peut être masqué par l'acidité due aux phosphates.

b) La solution moléculaire. - La faible teneur en lactose du colostrum qui explique la faible valeur de l'indice de réfraction 
du colostro-sérum, est compensée par une élévation de l'azote non protéique, dont la dissociation électrolytique est faible.

20. La solution colloïdale (colloïdes protéiniques et colloïdes minéraux).

Dans le lait cette solution contient deux sortes de colloides :

a) Les colloïdes protéiniques. - Ce sont :

L'albumine et la globuline qui, dispersées en petites particules, constituent des colloïdes moléculaires ;

La caséine qui existe sous la forme saline de caséinate de calcium. et affecte l'aspect de micelles d'assez grosses dimensions.

b) Les folloïdes minéraux qui se rámènent aux phosphates de calcium et un peu de phosphates magnésiens dont les micelles sont plus gros encore que ceux du caséinate.

Dans cet ensemble, les colloïdes moléculaires (albumine et globuline) sont les protecteurs des colloïdés salins (caséine et phosphates) contre lá coagulation provoquée par les acides et par la présure.

De même, le caséinate de calcium étant le plus stable est le colloïde protecteur du phosphate de calcium, colloïde protégé le moins stable en même temps qu'irréversible.

C'est ce que résumait Porcher en disant que " la chimie colloïdale du lait est dominée nettement par les phénomènes de protection, protection double, étayée, car si dans le complexe " caséinate de calcium + phosphates de calcium " celui-là protège celui-ci, le complexe lui-même sera protégé à son tour dans le lait par l'albumine et la globuline n.

Il est bien évident que la stabilité d'un tel ensemble colloïdal est d'autant plus grande que les colloïdes protecteurs sont en fortes proportions. C'est précisément ce qui a lieu dans le colostrum qui est riche en holoprotéides.

Encore faut-il distinguer parmi ceux-ci des différences. La globuline possède un pouvoír protecteur beaucoup plus élevé que la lactalbumine parce que sa fonction acide est plus marquée que sa fonction basique.

Le taux élevé de la globuline dans le colostrum protège donc considérablement les autres colloïdes, si bien que les réactions qu'il présenterait avec une grande netteté si la globuline était absente, peuvent ne pas se produire. C'est ce que nous examinerons plus loin.

$3^{0}$ L'émulsion (matière grasse).

Au même titre que le lait, le colostrum est une émulsion par la 
dispersion de la matière grasse qu'il renferme. Cependant les globules gras, sphériques, s'y trouvent sous deux états.

Les uns sont libres comme ceux du lait normal et leur diamètre oscille entre $1 \mu$ et $20 \mu$. Les autres sont inclus dans des cellules leucocytaires qui ont été décrites sous le nom de Corpuseules de Donne et dont les dimensions vont de 15 à $40 \mu$.

D'après OTT de VRIES [160] et REIssia [200], le nombre des gros globules est élevé par comparaison avec le lait normal.

En étudiant le degré de dispersion de la matière grasse, WEIGMANN [259] a trouvé que, jusqu'au sixième jour, ce degré est fortement modifié : le nombre des petits globules l'emporte sur celui des gros, tandis que le nombre de globules moyens est sensiblement le même que dans le lait.

(A suivre.)

\title{
LA GRADUATION DES BUTYROMÈTRES A CRÈ ME
}

UN NOUVEAU MODELLE DE BUTYROMETTRE

\author{
par \\ JEAN PIEN
}

Ingénieur Chimiste, Docteur ès Sciences, Directeur des Laboratoires de la Laiterie des Fermiers Réunis

Le problème qui se pose dans l'analyse industrielle des crèmes est le suivant :

Déterminer leur richesse et l'exprimer en grammes de matière grasse par kilogramme en effectuant l'analyse sur une prise d'essai pondérale (par exemple 5 grammes de crème).

Les raisons qui ont fait abandonner la prise d'essai volumétrique (par exemple $5 \mathrm{~cm}^{3}$ de crème) ainsi que l'expression des résultats par litre de crème sont trop connues pour qu'il soit nécessaire d'y insister de nouveau. Il est unanimement admis maintenant qu'il faut peser et non mesurer la prise d'essai et que le résultat doit être exprimé par kilogramme (ou pour 100 grammes) de crème.

La méthode d'analyse dite "Gerber-dilution» remplit automatiquement ces conditions puisqu'elle part de 10 grammes de erème dont on fait une dilution dans un volume final de $100 \mathrm{~cm}^{3}$. Cette dilution contient done 100 grammes de crème par litre. La méthode Gerber classique appliquée à cette dilution (comme dans le cas d'un lait) donne, avec un butyromètre à lait classique gradué au litre, la richesse par litre de cette dilution, c'est-à-dire le poids de matière grasse contenue dans les 100 grammes de crème que comporte un litre de cette dilution. En multipliant par 10 le chiffre lu au butyromètre on obtient donc la richesse de la crème en grammes de matière grasse par kilogramme. Cette méthode simple supprime 